\title{
应用纳米零价铁富集银的研究
}

\author{
顾天航 $a, b$ \\ 石君明 $a, b$ 滑熠龙 ${ }^{a, b}$ 刘静 ${ }^{a, b}$ \\ 王伟*,a,c \\ 张伟贤*,,$b$ \\ ( ${ }^{a}$ 污染控制与资源化研究国家重点实验室 上海 200092) \\ ( ${ }^{b}$ 同济大学环境科学与工程学院 上海 200092) \\ ( ${ }^{c}$ 同济大学化学科学与工程学院 上海 200092)
}

\begin{abstract}
摘要 大量研究表明, 纳米零价铁(nanoscale Zero-Valent Iron, nZVI)对水中重金属, 尤其是金、银等稀贵金属, 有良好的 分离富集作用. 利用纳米零价铁反应器证明了 $\mathrm{nZVI}$ 可从废水中分离低浓度的银离子 $\left(\mathrm{Ag}^{+}\right)$, 并生成高含量的 “银矿石”. 此外, 也证明了反应区氧化还原电位能够反映 $\mathrm{nZVI}$ 与 $\mathrm{Ag}^{+}$的反应速率和分离效率. 利用 $\mathrm{X}$ 射线衍射仪、 $\mathrm{X}$ 射线光电子 能谱和高分辨透射电子显微镜等手段对反应产物进行表征，证实了 $\mathrm{Ag}^{+}$可被 $\mathrm{nZVI}$ 还原为单质银，并以纳米颗粒的形式 $(<10 \mathrm{~nm}$ )沉积在 nZVI 表面. 与其他材料(常见吸附/还原材料)相比, nZVI 具有效率高, 受 $\mathrm{pH}$ 影响小的优点. 研究结果 表明, nZVI 是一种能够高效富集痕量银资源并产生高价值纳米银的材料.

关键词 纳米零价铁; 银; 富集; 纳米银; 纳米零价铁反应器
\end{abstract}

\section{Enrichment of Silver from Water Using Nanoscale Zero-Valent Iron (nZVI)}

\author{
Gu, Tianhang ${ }^{a, b}$ \\ Shi, Junming ${ }^{a, b}$ \\ Hua, Yilong ${ }^{a, b}$ \\ Liu, Jing ${ }^{a, b}$ \\ Zhang, Wei-xian*,a,b \\ ( ${ }^{a}$ State Key Laboratory of Pollution Control and Resource Reuse, Shanghai 200092) \\ ( ${ }^{b}$ College of Environmental Science and Engineering, Tongji University, Shanghai 200092) \\ ( ${ }^{c}$ School of Chemical Science and Engineering, Tongji University, Shanghai 200092)
}

Wang, Wei*,a,c

\begin{abstract}
Increasing evidence suggests that nanoscale zero-valent iron (nZVI) is an effective nanomaterial for the enrichment and separation of heavy metals from water, especially for recovering precious metals such as gold and silver from trace level sources. In this work, a nano-iron reactor, consisting of reaction zone, separation zone and reuse facilities, is applied to recovery of silver from aqueous solution using nZVI. We demonstrate that nZVI could sequester $\mathrm{Ag}^{+}(\mathrm{ca} .1 \mathrm{mg} / \mathrm{L})$ and be transformed into high-grade $(32.0 \mathrm{mg} / \mathrm{g})$ silver solids ("ore") as nZVI is recycled in this "reaction-separation-reuse" system. Besides, increasing hydraulic retention time (HRT), from $10 \mathrm{~min}$ to $60 \mathrm{~min}$, could enhance the enrichment efficiency and finally improve silver content in solid phase. We further demonstrate that there is a positive correlation between solution oxidation-reduction potential in reaction zone and $\mathrm{Ag}^{+}$concentration in effluent, and this relationship can be used to regulate the reaction kinetics and separation efficiency. Data from oxidation-reduction potential regulating experiment are presented and a mathematic formula is provided, proving this system is reliable and controllable. Solid phase characterizations with X-ray diffraction and X-ray photoelectron spectroscopy confirm that $\mathrm{Ag}^{+}$is reduced to metallic silver $\left(\mathrm{Ag}^{0}\right)$. Images acquired via high-resolution transmission electron microscopy reveal that $\operatorname{Ag}^{0}(<10 \mathrm{~nm})$ is deposited on the surface of $\mathrm{nZVI}(\mathrm{Ag}-\mathrm{nZVI})$. Pure silver nanoparticles (AgNPs, 9 32 nm) could be acquired by simply processing Ag-nZVI with sulfuric acid and polyvinyl pyrrolidone. Batch experiments confirm that $\mathrm{nZVI}$ is far more efficient and less $\mathrm{pH}$-dependent, comparing to other materials (e.g., mZVI, $\left.\alpha-\mathrm{Fe}_{2} \mathrm{O}_{3}, \mathrm{nTiO}_{2}\right) .99 \% \mathrm{Ag}^{+}(1000 \mathrm{mg} / \mathrm{L})$ could be sequestrated in less than $15 \mathrm{~s}$ with $1 \mathrm{~g} / \mathrm{L}$ nZVI. And the separation coefficient of $\mathrm{nZVI}$ for $\mathrm{Ag}^{+}$reaches $3.2 \times 10^{4}$, which is several orders of magnitude higher than that of conventional adsorbents and reductants $(102 \sim 741)$. This study demonstrates that $\mathrm{nZVI}$ is a powerful candidate to recover Ag from water (e.g., industrial wastewater, groundwater) with trace level silver and produce valuable AgNPs.

Keywords nanoscale zero-valent iron; silver; enrichment; silver nanoparticles; nano-iron reactor
\end{abstract}

\section{1 引言}

银(Silver, Ag)是一种具有银白色光泽的稀贵金属, 广泛地应用于流通货币、装饰品、工业催化、电子元件
制造等领域 ${ }^{[1]}$. 然而, 随着银需求不断增大及含银矿产 的大量开采，银资源紧缺问题正逐渐突显 ${ }^{[2,3]}$. 另一方 面, 含银废水(如冶炼废水、电镀废水)、废渣(如尾矿、 电子废弃物)等二次资源中的银未被有效回收利用，仅

*E-mail: 06ww@tongji.edu.cn; zhangwx@tongji.edu.cn

Received July 28, 2017; published September 18, 2017.

Supporting information for this article is available free of charge via the Internet at http://sioc-journal.cn.

Project supported by the National Postdoctoral Program for Innovative Talents (BX201700172) and the National Natural Science Foundation of China (No. 51578398).

项目受 “博新计划” (BX201700172)和国家自然科学基金(No. 51578398)资助. 
1997 年全球就有 $1.34 \times 10^{4} \mathrm{t}$ 银排入环境 ${ }^{[4 \sim 6]}$. 因此, 研 究如何从此类低浓度/低品位二次资源中有效分离/回收 银, 具有巨大的社会效益. 此外, 纳米银(Silver Nanoparticles, AgNPs) 由于具有优良的抗菌、催化及光学特性, 成为目前应用最广泛的纳米材料之一 ${ }^{[1,7,8]}$. 据统计, 全 球有 438 种在售商品含有纳米银, 占所有 “纳米” 商品 种数的 $24 \%(2015 \text { 年 })^{[9]}$. 如果能够以含银废水为原料, 生产高附加值的纳米银颗粒, 那么对经济与环境可持续 健康发展都将具有重要理论和现实意义.

近年来, 各国学者研究采用不同材料分离水中银离

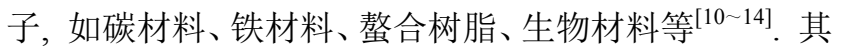
中, 纳米零价铁(nanoscale Zero-Valent Iron, nZVI)具有 独特 “核一壳” 结构, 内核为零价铁 $\left(\mathrm{Fe}^{0}\right)$, 外壳为铁(氢) 氧化物(如 $\mathrm{FeOOH}$ ), 具有尺寸小、比表面积大、反应活 性高、反应产物环境友好等特点 ${ }^{[15 ~ 17]}$. 近期研究表明, nZVI 可通过吸附、还原等作用有效分离水中金、银、 铀、硒等贵重(类)金属资源, 在重金属废水处理及资源 化方面表现出巨大应用潜力 ${ }^{[18 ~ 23]}$. 例如, 学者通过烧杯 实验考察 nZVI 富集银可行性及产物特征, 发现当初始 $\mathrm{Ag} / \mathrm{Fe}$ 反应质量比低于 1.25 时, nZVI 可将水中 $\mathrm{Ag}^{+}$还原 成纳米银颗粒 ${ }^{[18]}$; 另有学者采用介孔限域的方法将 nZVI 稳定结合于介孔碳管内并考察其对水中痕量金离 子 $(0.01 \mathrm{mg} / \mathrm{L})$ 富集能力, 发现 nZVI 可快速高效富集金 离子, 产生的金颗粒粒径仅为 $5 \mathrm{~nm}$, 可作为双金属催化 剂进一步回收再利用 ${ }^{[19]}$.

然而, 现有关于 $n Z V I$ 分离水中 $A g$ 等稀贵金属资源 的结果多来源于实验室烧杯(批次)试验, 尚缺乏对实际 应用过程中面临的反应器构型/设计、工艺参数选择及组 合优化、反应产物固液分离及回收等问题的研究和讨 论 $^{[18 ~ 23]}$. 近年来, 笔者所在研究团队通过 “小试一中试工程” 科学放大, 系统探索了 nZVI 连续处理实际重金 属废水可行性, 提出 “反应一分离一回用” 式连续流反应 器模型, 并成功将 nZVI 应用于含 $\mathrm{As} 、 \mathrm{Cu} 、 \mathrm{~Pb} 、 \mathrm{Zn}$ 、 $\mathrm{Ni}$ 等重金属的复杂冶炼废水处理工程 ${ }^{[24 ~ 26]}$. 在实践过 程中, 还发现实际废水中常存在多种低浓度稀贵金属离 子, 如某治炼废水中 $\mathrm{Ag}^{+}$浓度在 $0.12 \sim 10.54 \mathrm{mg} / \mathrm{L}$ 之间 波动(平均值为 $1.10 \mathrm{mg} / \mathrm{L}$ ). 通过文献检索, 关于 $\mathrm{nZVI}$ 连续富集回收废水中低浓度 $\mathrm{Ag}$ 的研究鲜有报道.

本文在团队前期研究基础上, 采用 “反应-分离-回 用” 式纳米零价铁反应器(Nano-Iron Reactor, NIR), 探讨 nZVI 连续富集水中低浓度 $\mathrm{Ag}^{+}$的工艺可行性, 考察了 相关反应条件影响及反应产物回收价值, 包括: (1)通过 连续流穿透实验研究不同水力停留时间(Hydraulic Retention Time, HRT)对低浓度 $\mathrm{Ag}^{+}$(约 $1 \mathrm{mg} / \mathrm{L}$ ) 富集效果的 影响, 分析反应区氧化还原电位 $\left(E_{\mathrm{h}}\right)$ 与 $\mathrm{Ag}$ 富集效果间 相关性并进行稳态实验验证; (2) 通过批次实验探究 nZVI 对银富集性能及关键因素影响, 结合多种固相表 征手段进一步证明反应机理及 $\mathrm{Ag}$ 回收价值. 本研究将
为 $\mathrm{nZVI}$ 富集痕量 $\mathrm{Ag}$ 的应用提供理论依据和指导.

\section{2 结果与讨论}

\section{1 连续流实验}

本文首先探讨了 NIR 富集 $\mathrm{Ag}^{+}$的可行性及可控性. NIR 由反应区、分离区及回流装置三部分组成(图 1, 表 $\mathrm{S} 1)$ ：其中反应区为全混合搅拌釜式反应器，具有连续 处理废水并有一定抗冲击能力的特点; 由于 nZVI 自身 具有磁性，颗粒间因为偶极间作用会形成链状团聚体， 在反应器中这些链状团聚物会进一步形成微米级团聚 体, 平均粒径约 $7 \mu \mathrm{m}$, 尺寸较小, 因此简单的机械搅拌 即可达到混合均匀的目的; 进水 $\left(\left[\mathrm{Ag}^{+}\right]_{\text {in }}=1 \mathrm{mg} / \mathrm{L}\right)$ 由蠕 动百连续输入反应区与 $\mathrm{nZVI}$ 充分混合均匀并反应, 而 后混合液流入分离区; 分离区为一坚流式沉淀池，利于 $\mathrm{nZVI}$ 在底部雉形泥斗中沉积浓缩和回流, 根据笔者所 在团队前期研究, 当表面负荷为 $0.5 \mathrm{~m}^{3} /\left(\mathrm{m}^{2} \bullet \mathrm{h}\right.$ ) 时(略低于 本文最大表面负荷), $92 \%$ 的 nZVI 可后经重力沉降实现 固液分离，分离后上清液通过分离区上部三角堰连续流 出(出水); 沉积在分离区底部的 nZVI 经蠕动泵回流至 反应区继续参与反应, 以提高 nZVI 利用率 ${ }^{[27]}$.

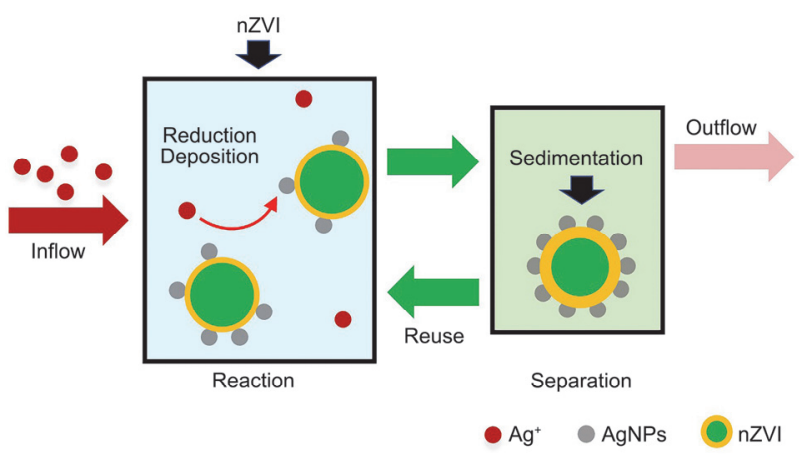

图 1 纳米零价铁连续流反应器富集银示意图

Figure 1 The scheme of silver enrichment with nZVI reactor

\subsection{1 连续流穿透实验}

本部分实验研究了 NIR 在不同 HRT 的条件下(表 S2)对 $\mathrm{Ag}^{+}$的富集性能. 以钠离子 $\left(\mathrm{Na}^{+}\right)$作为示踪剂比较 反应器对 $\mathrm{Ag}^{+}$的富集效果, 并监测反应区 $E_{\mathrm{h}}$ 的变化, 研 究 $E_{\mathrm{h}}$ 与 $\mathrm{Ag}^{+}$富集效率的关系.

当 $\mathrm{HRT}=10 \mathrm{~min}$ 时(图 2A), 出水中 $\mathrm{Na}^{+}$浓度快速上 升, $2 \mathrm{~h}$ 后完全穿透 $\left(\left[\mathrm{Na}^{+}\right]_{\text {出水 }}=\left[\mathrm{Na}^{+}\right]_{\text {进水 }}\right)$, 而 $9.5 \mathrm{~h}$ 后 $\mathrm{Ag}^{+}$ 浓度仍低于原水浓度的 $20 \%$. 可见 $\mathrm{nZVI}$ 对 $\mathrm{Ag}^{+}$具有极 强的截留能力(截留率 $>80 \%$ ). 当系统 HRT 为 30 或 60 $\min$ (图 $2 \mathrm{~B} \sim 2 \mathrm{C}$ ) 时, 反应器对 $\mathrm{Ag}^{+}$富集能力提高. 在累 积进入系统 $\mathrm{Ag}$ 量不变的情况下, 当 HRT 由 $10 \mathrm{~min}$ 延长 至 $60 \mathrm{~min}, \mathrm{Ag}^{+}$最大穿透率由 $19.0 \%$ 降至 $4.1 \%$, 这可能是 因为 HRT 的延长增加了 $\mathrm{Ag}^{+}$与 $\mathrm{nZVI}$ 的接触时间, 使反 应更加充分.

不同 HRT 条件下, 反应区 $E_{\mathrm{h}}$ 和出水中 $\mathrm{Ag}^{+}$浓度均 
存在一定相关性(图 2A $\sim 2 \mathrm{C}$ ). 例如 $\mathrm{HRT}=10 \mathrm{~min}$ 时, 出 水中 $\mathrm{Ag}^{+}$浓度和反应区内 $E_{\mathrm{h}}$ 的变化趋势可分为两个阶 段: 阶段 $1(0 \sim 5.5 \mathrm{~h}) E_{\mathrm{h}}$ 快速上升, 出水 $\mathrm{Ag}^{+}$浓度稳定在 $40 \mu \mathrm{g} / \mathrm{L}$ 以下 $\left(C / C_{0}<0.04\right)$; 阶段 $2(5.5 \sim 9.5 \mathrm{~h}) E_{\mathrm{h}}$ 维持稳 定, 出水 $\mathrm{Ag}^{+}$浓度快速上升. 比较两阶段终点 $C / C_{0}$ 值, 分别由 0.035 迅速增加至 $0.194(\mathrm{HRT}=10 \mathrm{~min}), 0.013$ 至 $0.057(\mathrm{HRT}=30 \mathrm{~min}), 0.011$ 至 $0.028(\mathrm{HRT}=60 \mathrm{~min})$. 因 此, 在实际应用过程中, 根据 $E_{\mathrm{h}}$ 与出水 $\mathrm{Ag}^{+}$浓度的关系, 可将 $E_{\mathrm{h}}$ 作为在线反映出水 $\mathrm{Ag}^{+}$浓度的参数, 根据 $E_{\mathrm{h}}$ 的 变化趋势决定投加新鲜 nZVI 或排出分离区固相产物的 时间点.

以 $\mathrm{HRT}=10 \mathrm{~min}$ 时的结果为例(图 $2 \mathrm{~A}), E_{\mathrm{h}}$ 和出水 $\mathrm{Ag}^{+}$浓度变化的原因可能是: (1)阶段 1 过程中, 分离区 表面水力负荷 $\left[0.566 \mathrm{~m}^{3} /\left(\mathrm{m}^{2} \cdot \mathrm{h}\right)\right]$ 大于 $\mathrm{nZVI}$ 颗粒有效分离 的极限表面水力负荷 $\left[0.100 \mathrm{~m}^{3} /\left(\mathrm{m}^{2} \bullet \mathrm{h}\right)\right]$, 部分 $\mathrm{nZVI}$ 随出 水流失, 因而 $E_{\mathrm{h}}$ 上升速度快, 同时反应区较高浓度的 $\mathrm{nZVI}$ 促进 $\mathrm{Ag}^{+}$被快速稳定富集; 阶段 2 过程中反应区内 $\mathrm{nZVI}$ 浓度较低, nZVI 随出水流失量减少, 因此 $E_{\mathrm{h}}$ 保持 相对稳定, 反应区 $n Z V I$ 浓度过低导致系统对 $\mathrm{Ag}^{+}$的富

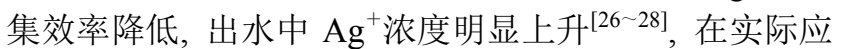
用中需要添加新鲜 nZVI; (2)阶段 $1 \mathrm{nZVI}$ 颗粒中 $\mathrm{Fe}^{0}$ 含量 较高, 还原能力较强, 可快速富集 $\mathrm{Ag}^{+}$, 而在阶段 2 , nZVI 经过在较长时间的循环使用中不断受到 $\mathrm{H}_{2} \mathrm{O}$ 、混
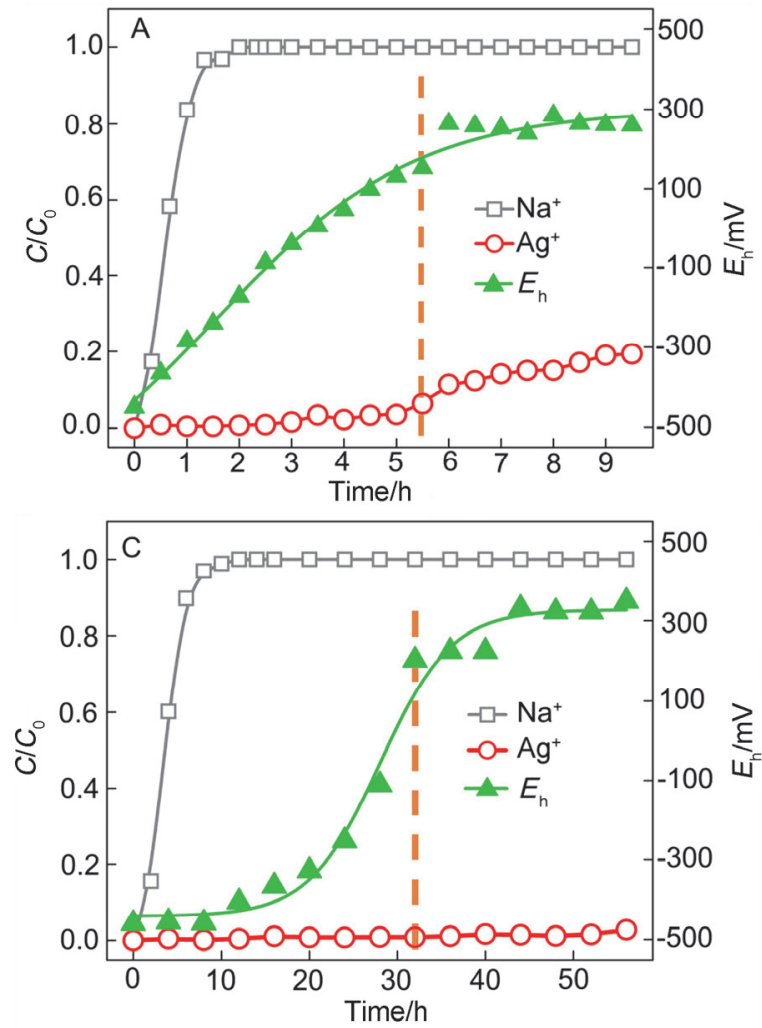

图 2 不同水力停留时间条件下 $\mathrm{Ag}^{+}$富集情况、出水中 $\mathrm{Na}^{+}$浓度变化及反应区 $E_{\mathrm{h}}$ 变化: (A) HRT=10 min; (B) HRT=30 min; (C) HRT=60 min; (D) 平均富集率; (E)反应产物银含量

Figure 2 Effluent concentrations of $\mathrm{Ag}^{+}$and $\mathrm{Na}^{+}$, and variation of $E_{\mathrm{h}}$ as a function of reaction time under different: (A) HRT $=10 \mathrm{~min}$; (B) HRT=30 $\mathrm{min}$; (C) HRT $=60 \mathrm{~min}$; (D) average silver enrichment ratio; (E) silver content in final products

Reaction conditions: $\mathrm{nZVI}$ dosage $=0.5 \mathrm{~g},\left[\mathrm{Ag}^{+}\right]_{\mathrm{in}}=1.0 \mathrm{mg} / \mathrm{L}$, initial $\mathrm{pH}=7.0,25{ }^{\circ} \mathrm{C}$

杂的 $\mathrm{O}_{2}$ 及溶液中 $\mathrm{NO}_{3}^{-}$的腐蚀, $\mathrm{Fe}^{0}$ 含量降低, 还原能力 减弱，水质开始恶化，因此在实际应用中需在适当时间 排出反应后固体颗粒.

根据物料守恒计算, 可知在不同 HRT 条件下(处理 $17.1 \mathrm{~L}$ 含银原水), $\mathrm{nZVI}$ 对 $\mathrm{Ag}^{+}$的平均富集率为: $93.3 \%$ $(10 \mathrm{~min})<98.6 \%(30 \mathrm{~min})<99.5 \%$ (60 min), 再一次证 明延长 HRT 可提高 nZVI 的富集效率(图 2D); 当 HRT 由 $10 \mathrm{~min}$ 增加至 $30 \mathrm{~min}$ 时，平均富集率提高了 $5.3 \%$, 而由 $30 \mathrm{~min}$ 至 $60 \mathrm{~min}$ 时，平均富集率仅提高 $0.9 \%$, 综 合考虑富集率及处理效率, HRT 设为 $10 \mathrm{~min}$ 或 $30 \mathrm{~min}$ 较合适. 此外, 还可计算出 NIR 内反应产物(Ag-nZVI) 上银含量分别为 $21.5(10 \mathrm{~min}) 、 30.2(30 \mathrm{~min}) 、 32.0(60$ $\mathrm{min}) \mathrm{mg} / \mathrm{g}$ (图 2E). 考虑到 nZVI 会腐蚀产生(氢)氧化物, 实际银含量会略低于上述三个值, 但仍高于高品位银矿 石 $(6 \sim 10 \mathrm{mg} / \mathrm{g})^{[29]}$, 表明利用 nZVI 可将分散的低品位的 银资源富集，并浓缩至具有较高回收价值的水准.

\subsection{2 $E_{\mathrm{h}}$ 稳态调控实验}

依据 2.1.1 中出水 $\mathrm{Ag}^{+}$浓度和反应区 $E_{\mathrm{h}}$ 的联系, 可 得出以下结论: $E_{\mathrm{h}}$ 变化趋势能够反映 $\mathrm{nZVI}$ 对 $\mathrm{Ag}^{+}$的富集 效率. 为了进一步验证这种规律，模拟了向 NIR 内间歇 性投加新鲜 nZVI 时系统运行的稳定性. 反应开始前向 反应区投加 $0.5 \mathrm{~g}$ 新鲜 $\mathrm{nZVI}$, 之后每次 $E_{\mathrm{h}}$ 趋于稳定时投 加 $0.1 \mathrm{~g}$ 新鲜 $\mathrm{nZVI}$ (投加 3 次).
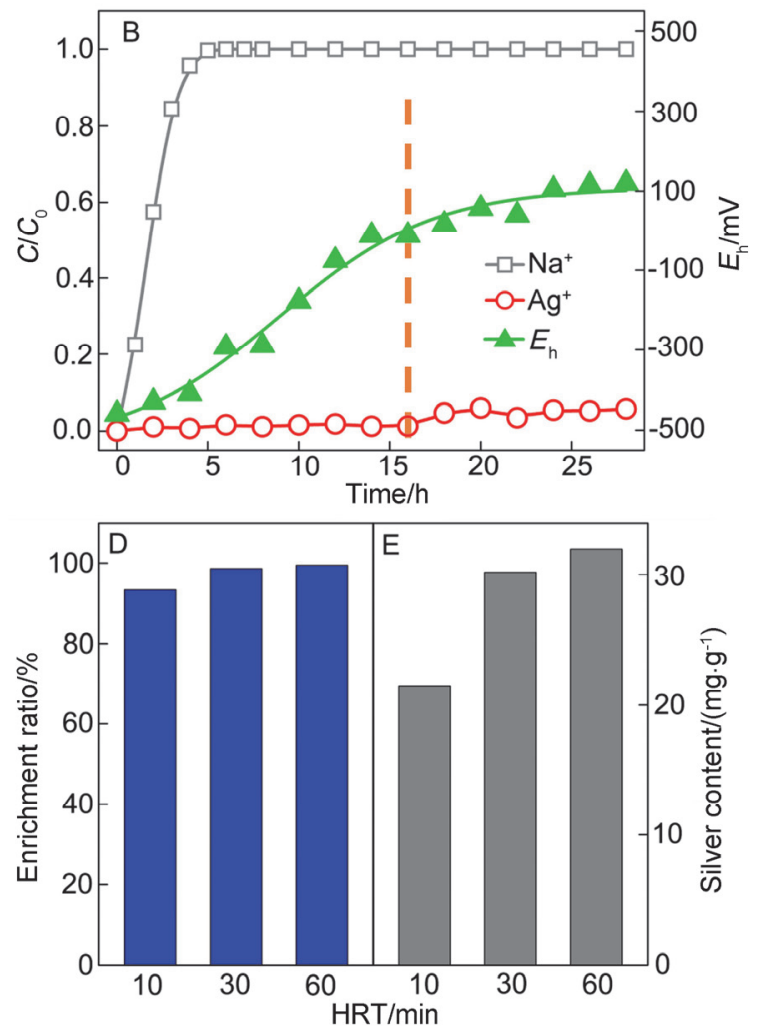
图 3A、3B 反映了出水 $\mathrm{Ag}^{+}$浓度、反应区 $E_{\mathrm{h}}$ 的变化. 实验结果表明, 连续流穿透实验中 $E_{\mathrm{h}}$ 和出水 $\mathrm{Ag}^{+}$浓度的 变化趋势得到多次重现. 每次反应进行到一定程度, 随 着 $\mathrm{nZVI}$ 的流失及氧化, 反应区内 $\mathrm{Fe}^{0}$ 浓度降低, $E_{\mathrm{h}}$ 由约 $-500 \mathrm{mV}$ 上升至约 $300 \mathrm{mV}$ 并趋于平稳, 对 $\mathrm{Ag}^{+}$的富集 能力会逐渐减弱, 出水中 $\mathrm{Ag}^{+}$浓度由 $3 \mu \mathrm{g} / \mathrm{L}$ 上升至 165 $\mu \mathrm{g} / \mathrm{L}$; 补充 $0.1 \mathrm{~g}$ 新鲜 $\mathrm{nZVI}$ 后, 反应区 $\mathrm{Fe}^{0}$ 浓度显著提 高, $E_{\mathrm{h}}$ 迅速降低至 $-350 \mathrm{mV}$, NIR 对 $\mathrm{Ag}^{+}$的富集能力提 高, 出水中 $\mathrm{Ag}^{+}$降低至 $4 \mu \mathrm{g} / \mathrm{L}$.

图 $3 \mathrm{C}$ 结果表明: 反应区 $E_{\mathrm{h}}$ 与出水中 $\mathrm{Ag}^{+}$浓度的对

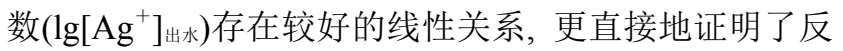
应区 $E_{\mathrm{h}}$ 能够反映 NIR 对 $\mathrm{Ag}^{+}$的富集性能. 以上结果表 明: 可利用反应区 $E_{\mathrm{h}}$ 与出水中 $\mathrm{Ag}^{+}$浓度之间的联系, 建 立 $E_{\mathrm{h}}$ 调控机制, 以此来调控 NIR 富集效率.
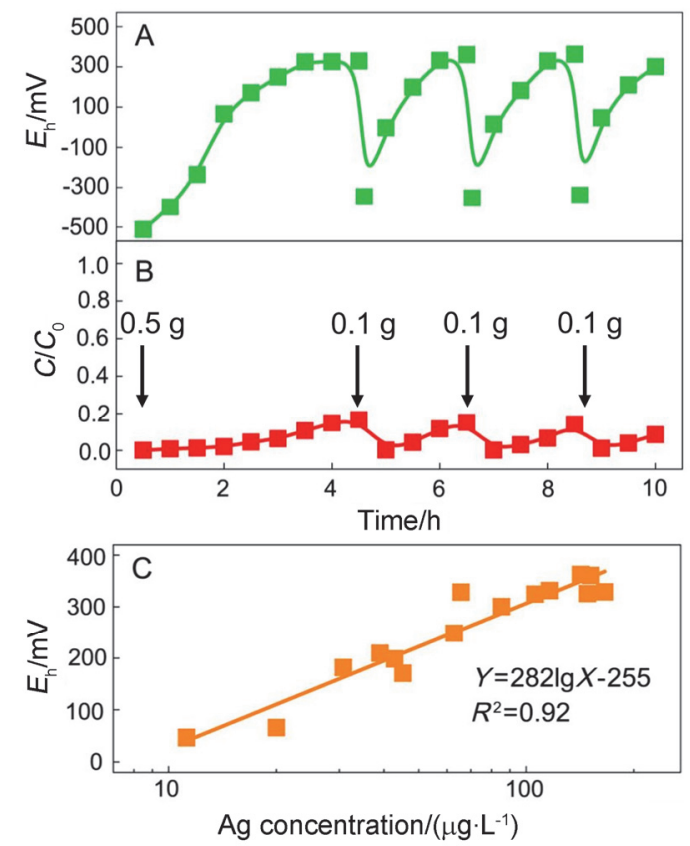

图 $3 E_{\mathrm{h}}$ 稳态调控实验结果: $(\mathrm{A})$ 反应区 $E_{\mathrm{h}}$ 变化; (B) 出水 $\mathrm{Ag}^{+}$浓度变化; (C)反应区 $E_{\mathrm{h}}$ 和出水 $\mathrm{Ag}^{+}$浓度的相关性

Figure 3 Results of $E_{\mathrm{h}}$ regulating experiments: (A) changes of $E_{\mathrm{h}}$ in reaction zone; (B) $\mathrm{Ag}^{+}$concentration in effluent; (C) relationship between $E_{\mathrm{h}}$ and $\mathrm{Ag}^{+}$concentration in effluent

Reaction conditions: $\mathrm{HRT}=10 \mathrm{~min},\left[\mathrm{Ag}^{+}\right]_{\mathrm{in}}=1.0 \mathrm{mg} / \mathrm{L}$, initial $\mathrm{pH}=7.0$, $25{ }^{\circ} \mathrm{C}$

\section{2 批次实验}

\subsection{1 不同材料对 $\mathrm{Ag}^{+}$的富集}

为了研究 nZVI 富集 $\mathrm{Ag}^{+}$的能力, 本部分实验选取 与 nZVI 尺寸或组分相同的三种材料 [三氧化二铁 $\left(\alpha-\mathrm{Fe}_{2} \mathrm{O}_{3}\right)$; 纳米二氧化钛 $\left(\mathrm{nTiO}_{2}\right)$; 微米零价铁 $\left.(\mathrm{mZVI})\right]$, 比较其对溶液中 $\mathrm{Ag}^{+}(\mathrm{pH}=5)$ 的富集效果. 实验结果如 图 4A 所示, nZVI、mZVI、 $\alpha-\mathrm{Fe}_{2} \mathrm{O}_{3} 、 \mathrm{nTiO}_{2}$ 对 $\mathrm{Ag}^{+}$的富 集率分别为 $99.9 \% 、 26.3 \% 、 12.8 \%$ 和 $10.3 \%$.
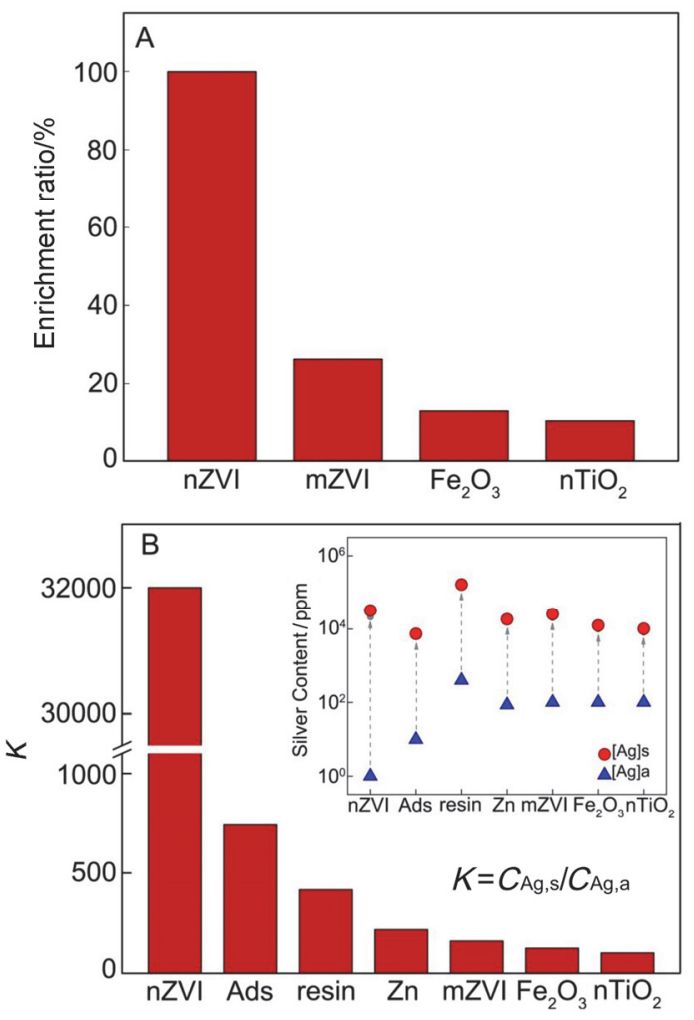

图 4 不同材料对 $\mathrm{Ag}^{+}$富集效果: (A)总体富集率; (B)分离系数; 插图 为富集前后 $\mathrm{Ag}$ 在水相和固相上含量变化

Figure 4 Enrichment performances of $\mathrm{Ag}^{+}$by different materials: (A) total enrichment ratio; (B) separation coefficient; Inset: $\mathrm{Ag}$ content in liquid and solid phase

Reaction conditions: material dosage $=1.0 \mathrm{~g} / \mathrm{L},\left[\mathrm{Ag}^{+}\right]_{0}=100 \mathrm{mg} / \mathrm{L}$, initial $\mathrm{pH}$ $=5.0$, rotating speed $=200 \mathrm{r} / \mathrm{min}$, reaction time $=4 \mathrm{~h}, 25{ }^{\circ} \mathrm{C}$

上述结果的原因可能是: (1) 溶液中 nZVI、mZVI、 $\alpha-\mathrm{Fe}_{2} \mathrm{O}_{3} 、 \mathrm{nTiO}_{2}$ 颗粒表面吸附位点(-OH)通过吸附作用捕 获 $\mathrm{Ag}^{+} ;(2) \alpha-\mathrm{Fe}_{2} \mathrm{O}_{3}$ 和 $\mathrm{nTiO}_{2}$ 仅能通过表面有限的活性位 点 $(-\mathrm{OH})$ 吸附溶液中少量 $\mathrm{Ag}^{+}$; 而 nZVI、mZVI 的主要成 分零价铁 $\left(\mathrm{Fe}^{0}\right)$ 与 $\mathrm{Ag}^{+}$发生氧化还原反应[式(1)]:

$$
2 \mathrm{Ag}^{+}+\mathrm{Fe}^{0} \rightarrow 2 \mathrm{Ag}^{0}+\mathrm{Fe}^{2+} \Delta E^{\theta}=1.21 \mathrm{~V}
$$

$n Z V I$ 和 mZVI 可通过还原作用强化对 $\mathrm{Ag}^{+}$的富集; (3) nZVI 比表面积可达 $30 \mathrm{~m}^{2} / \mathrm{g}$, 而 mZVI 仅 $0.01 \sim 1$ $\mathrm{m}^{2} / \mathrm{g}^{[30]}$, 在相同投加量情况下, nZVI 具有更多活性位点, 反应活性更强; (4) mZVI 与 $\mathrm{Ag}^{+}$反应过程中产生的铁氧 化物沉积在颗粒表面，降低了 $\mathrm{Fe}^{0}$ 与 $\mathrm{Ag}^{+}$之间的电子传 递效率, 阻碍反应的进一步进行 ${ }^{[31,32]}$, 而 nZVI 壳层存 在缺陷，可作为电子传递通道，整个壳层可视为半导体， 因此效率受表面氢氧化物沉积影响不明显.

由图 4B 可见, nZVI 对 $\mathrm{Ag}^{+}$的分离系数 $\left(K=10^{4.51}\right.$ $\mathrm{L} / \mathrm{kg}$ ) 比其它材料 $\left(K: 10^{2.01} \sim 10^{2.87} \mathrm{~L} / \mathrm{kg}\right)$ 高 $1 \sim 3$ 个数量 级 ${ }^{[33-35]}$, 可将水相中低浓度的 $\mathrm{Ag}^{+}$富集至固相. 实验结 果表明, nZVI 可高效、快速富集溶液中的 $\mathrm{Ag}^{+}$.

上述实验结果表明, nZVI 是一种高效富集 $\mathrm{Ag}^{+}$的材 料, 可通过吸附、还原等作用将溶液中 $\mathrm{Ag}^{+}$高效、快速 
富集. 既能回收废水中的银资源, 也能保证出水水质, 减少银对生态系统及人体健康造成的危害.

\subsection{2 初始 $\mathrm{pH}$ 对 $\mathrm{Ag}^{+}$富集的影响}

溶液 $\mathrm{pH}$ 影响颗粒表面电荷分布、离子形态, 进而 影响固相颗粒表面离子的传质过程. 因此, 本工作研究 了溶液初始 $\mathrm{pH}(3 \sim 9)$ 对 nZVI、mZVI 富集 $\mathrm{Ag}^{+}$的影响. 实验结果表明，不同溶液初始 $\mathrm{pH}$ 条件下, nZVI 对 $\mathrm{Ag}^{+}$ 的富集率均大于 $99 \% ; \mathrm{mZVI}$ 对 $\mathrm{Ag}^{+}$的富集率由 $63.9 \%$ $(\mathrm{pH}=3)$ 降至 $26.3 \%(\mathrm{pH}=5)$, 又上升至 $83.3 \%(\mathrm{pH}=9)$ (图 5).

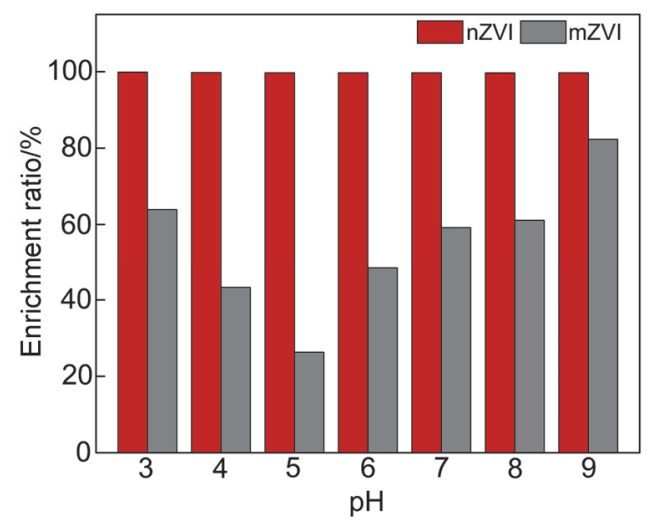

图 5 溶液初始 $\mathrm{pH}$ 对 $\mathrm{Ag}^{+}$富集率的影响

Figure 5 Effect of initial $\mathrm{pH}$ toward $\mathrm{Ag}^{+}$enrichment

Reaction conditions: material dosage $=1.0 \mathrm{~g} / \mathrm{L},\left[\mathrm{Ag}^{+}\right]_{0}=100 \mathrm{mg} / \mathrm{L}$, rotating speed $=200 \mathrm{r} / \mathrm{min}$, reaction time $=4 \mathrm{~h}, 25{ }^{\circ} \mathrm{C}$

在 $\mathrm{pH}$ 为 $3 \sim 9$ 的 $\mathrm{AgNO}_{3}$ 溶液中, 银以 $\mathrm{Ag}^{+}$的形式 存在. nZVI 和 $\mathrm{mZVI}$ 表面等电点约为 $8.3^{[15]}$, 因此当溶液 $\mathrm{pH}<8.3$ 时, 颗粒表面以正电荷为主, 不利于对 $\mathrm{Ag}^{+}$的 吸附; 当 $\mathrm{pH}>8.3$ 时, 颗粒表面带负电荷, 促进对 $\mathrm{Ag}^{+}$ 的吸附.

在当前反应条件下 $(n Z V I$ 或 $m Z V I$ 投加量 $=1 \mathrm{~g} / \mathrm{L}$, $\left.\left[\mathrm{Ag}^{+}\right]=100 \mathrm{mg} / \mathrm{L}\right), \mathrm{nZVI}$ 比表面积大, 反应活性高, 投 加至溶液(初始 $\mathrm{pH}$ 3 9) 中会迅速与 $\mathrm{H}_{2} \mathrm{O}$ 等物质反应, 产生 $\mathrm{OH}^{-}$, 使溶液 $\mathrm{pH}$ 快速稳定在 $7.5 \sim 9.5$ 范围内 ${ }^{[15]}$, 并且 $n Z V I$ 主要通过还原作用 $\left(\mathrm{Ag}^{+} \rightarrow \mathrm{Ag}\right)$ 富集溶液中的 $\mathrm{Ag}^{+}$, 体系具有强还原性, 因此富集结果受 $\mathrm{pH}$ 影响微 弱. mZVI 主要通过吸附、还原作用富集 $\mathrm{Ag}^{+}$. 低 $\mathrm{pH}$ 条 件下, mZVI 表面带正电, 但表面钝化层脱落后裸露出 的新鲜零价铁具有较强还原作用, 促进颗粒对 $\mathrm{Ag}^{+}$的富 集, 因此低 $\mathrm{pH}$ 利于 $\mathrm{Ag}^{+}$的去除; 高 $\mathrm{pH}$ 条件下, mZVI 表面钝化层负电荷比例提高后吸附作用增强, 进而对 $\mathrm{Ag}^{+}$的去除率提高. 实验结果表明, 在当前实验条件下, $\mathrm{nZVI}$ 富集 $\mathrm{Ag}^{+}$的过程基本不受溶液初始 $\mathrm{pH}$ 影响.

\subsection{3 nZVI 投加量的影响}

不同 nZVI 投加量的条件下, $\mathrm{Ag}^{+}$富集动力学过程如 图 6 所示. 投加 $0.10 \mathrm{~g} / \mathrm{L} \mathrm{nZVI}$ 时, $1 \mathrm{~min}$ 内 $\mathrm{Ag}^{+}$被快速分
离且溶液中 $\mathrm{Ag}^{+}$浓度到平衡, $30 \mathrm{~min}$ 时 $\mathrm{Ag}^{+}$富集率为 $53 \%$. 当 nZVI 浓度增加至 $0.25 、 0.50$ 和 $1.00 \mathrm{~g} / \mathrm{L}$ 时，富 集 $99 \%$ 以上 $\mathrm{Ag}^{+}$所需时间分别为 $5 \mathrm{~min} 、 15 \mathrm{~s}$ 和 $10 \mathrm{~s}$. 实 验结果表明, nZVI 对 $\mathrm{Ag}^{+}$有强富集能力, 且提高 nZVI 投加量时, nZVI 颗粒中对 $\mathrm{Ag}^{+}$的吸附位点增多, 加快了 $\mathrm{nZVI}$ 颗粒 $\mathrm{Ag}^{+}$富集速率.

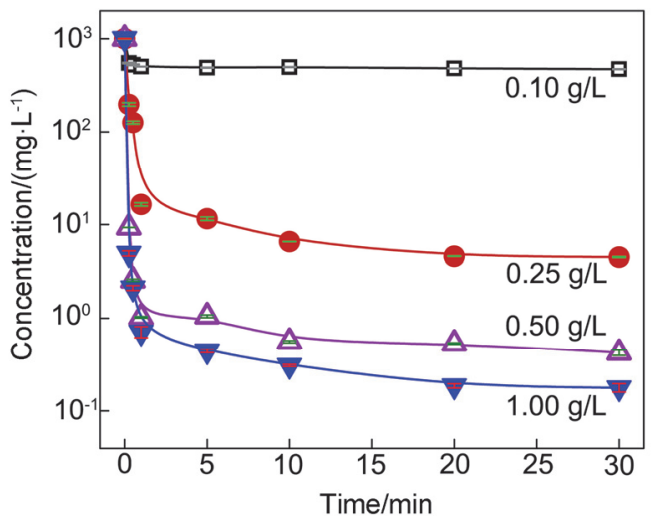

图 $6 \mathrm{nZVI}$ 投加量对 $\mathrm{Ag}^{+}$富集效果的影响

Figure 6 Effect of nZVI dosage toward $\mathrm{Ag}^{+}$enrichment

Reaction conditions: $\left[\mathrm{Ag}^{+}\right]_{0}=1000 \mathrm{mg} / \mathrm{L}$, initial $\mathrm{pH}=5.0$, rotating speed $=$ $200 \mathrm{r} / \mathrm{min}, 25{ }^{\circ} \mathrm{C}$

图 $\mathrm{S} 1$ 为不同初始水质负荷 $(\mathrm{Ag} / \mathrm{nZVI}, \mathrm{m} / \mathrm{m})$ 条件下 $\mathrm{nZVI}$ 对 $\mathrm{Ag}^{+}$的富集能力. 当水质负荷为 $0.05 \sim 4.50$ $\mathrm{g}-\mathrm{Ag}^{+} / \mathrm{g}-\mathrm{nZVI}$ 时, nZVI 对 $\mathrm{Ag}^{+}$的富集能力呈线性递增趋 势; 当负荷大于 $4.50 \mathrm{~g}-\mathrm{Ag}^{+} / \mathrm{g}-\mathrm{nZVI}$ 时, $\mathrm{nZVI}$ 对 $\mathrm{Ag}^{+}$的富 集能力趋于饱和, 达到最大值 $5.30 \mathrm{~g}-\mathrm{Ag} / \mathrm{g}-\mathrm{nZVI}$. nZVI 主要成分 $\mathrm{Fe}^{0}$ 具有较强的还原能力, 可还原 $\mathrm{Ag}^{+}$[式(1)], 反应产物结构态亚铁 $\left(\mathrm{Fe}^{2+}\right)$ 可还原 $\mathrm{Ag}^{+}$[式(2)]:

$$
\mathrm{Fe}^{2+}+\mathrm{Ag}^{+} \rightarrow \mathrm{Fe}^{3+}+\mathrm{Ag}^{0}
$$

因此 $1 \mathrm{~mol} / \mathrm{L} \mathrm{Fe}^{0}$ 可还原 $3 \mathrm{~mol} / \mathrm{L} \mathrm{Ag}^{+}$, 计算可得其 理论最大富集能力可达 $5.78 \mathrm{~g}-\mathrm{Ag} / \mathrm{g}-\mathrm{nZVI}$. 实际反应过 程中, nZVI 中还原组分 $\left(\mathrm{Fe}^{0}\right.$ 、结构态亚铁)会受到 $\mathrm{H}_{2} \mathrm{O}$ 及 其它氧化性组分 $\left(\mathrm{O}_{2}\right.$ 及 $\mathrm{NO}_{3}^{-}$等 $)$的影响, 实际最大富集 能力为 $5.30 \mathrm{~g}-\mathrm{Ag} / \mathrm{g}-\mathrm{nZVI}$, 略低于理论值.

\section{3 产物固相表征}

图 $7 \mathrm{~A}$ 为新鲜 $\mathrm{nZVI}$ 及其与 $\mathrm{Ag}^{+}$反应后固相产物 (Ag-nZVI) 的 XRD 谱图: nZVI 在 $2 \theta=44.9^{\circ}$ 存在一个弱 且宽化的衍射峰, 为 $\alpha-\mathrm{Fe}(0)$. 而反应产物在 $2 \theta=37.9^{\circ}$ 、 $44.1^{\circ} 、 64.2^{\circ} 、 77.2^{\circ}$ 和 $81.3^{\circ}$ 产生较为尖锐的衍射峰, 与 $\mathrm{Ag}^{0}$ 的(JCPDS 65-2871)标准衍射图谱相吻合, 表明 $\mathrm{Ag}^{+}$ 被 $\mathrm{nZVI}$ 还原为单质银 $\left(\mathrm{Ag}^{0}\right)$. 为进一步确定 $\mathrm{nZVI}$ 与 $\mathrm{Ag}^{+}$反应后 $\mathrm{Ag}$ 元素的价态, 对反应后的固体进行了高 分辨 XPS 测定, 图 7B 为产物表面 $\mathrm{Ag} 3 \mathrm{~d}$ 的图谱, 两个 峰 $\mathrm{Ag} 3 \mathrm{~d}_{3 / 2}$ 和 $\mathrm{Ag} 3 \mathrm{~d}_{5 / 2}$ 的结合能位于 $367.9 \mathrm{eV}$ 与 373.9 $\mathrm{eV}$, 此结果与 Zhang 等 ${ }^{[18]}$ 研究相一致, 再次证明 $\mathrm{nZVI}$ 表面银元素为 $\mathrm{Ag}^{0}$. 

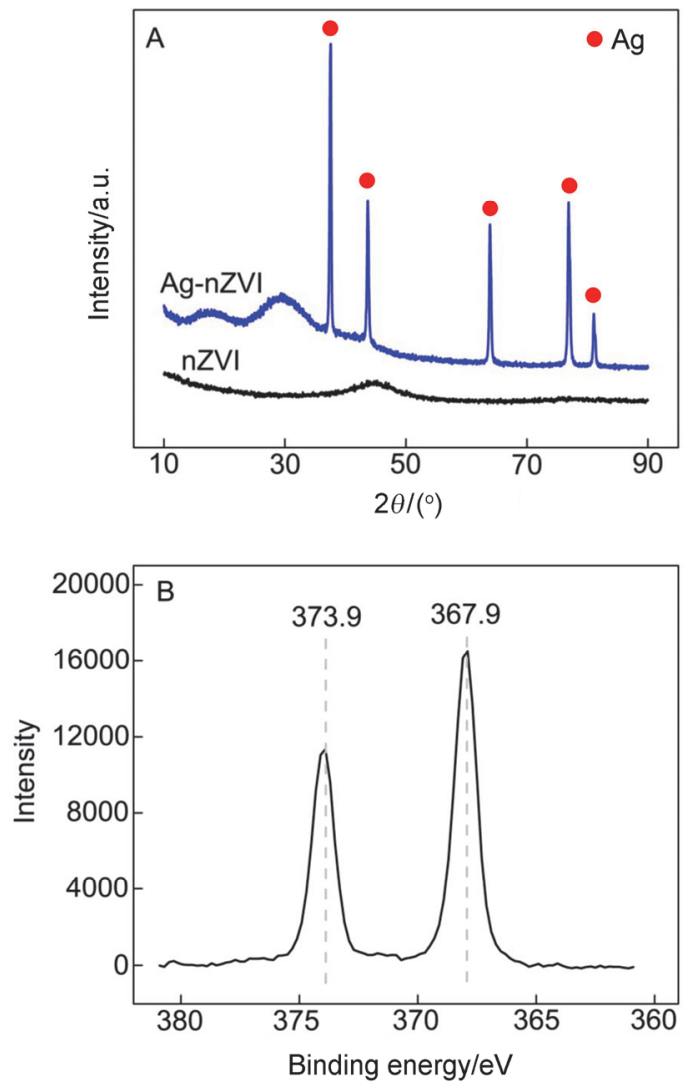

图 7 (A) nZVI 反应前后 XRD 谱图; (B)反应产物 $\mathrm{Ag} 3 \mathrm{~d}$ XPS 谱图

Figure 7 (A) XRD patterns of fresh and spent nZVI; (B) XPS spectrum of $\mathrm{Ag} 3 \mathrm{~d}$

Reaction conditions: $\left[\mathrm{Ag}^{+}\right]_{0}=1000 \mathrm{mg} / \mathrm{L}, \mathrm{nZVI}$ dosage $=1.0 \mathrm{~g} / \mathrm{L}$, initial $\mathrm{pH}=$ 5.0 , rotating speed $=200 \mathrm{r} / \mathrm{min}, 25{ }^{\circ} \mathrm{C}$

反应产物中单质银物相、元素价态确定之后，通过 HR-TEM 研究产物形貌结构. 图 S2 为新鲜 nZVI, 呈链 球状, 粒径约为 $100 \mathrm{~nm}$, 具有明显 “核一壳” 结构, 表面 氧化物层厚度约 $3 \mathrm{~nm}$. 图 $8 \mathrm{~A}$ 和图 $8 \mathrm{~B}$ 为反应后的 Ag-nZVI，可发现产物颗粒表面附着有直径 4 10 nm 球 形颗粒. 使用聚乙烯吡咯烷酮 K-30(PVP)和稀硫酸处理 后 ${ }^{[36]}, \mathrm{nZVI}$ 载体被除去, 球形颗粒从载体上分离, 分布 均匀(图 8C). 对图中 100 个纳米颗粒粒径进行统计: 粒 径范围 9 32 nm, 平均值 $16.4 \mathrm{~nm}$. 单个纳米颗粒(图 $8 \mathrm{D})$ 为球形, 直径约 $10 \mathrm{~nm}$, 晶面间距 $(2.35 \AA)$ 与银单质 相吻合, 进一步证实了反应产物为 AgNPs. 反应产物 Ag-nZVI 的 Ag-Fe 双金属结构可形成原电池, 可能可作 为负载型 $\mathrm{AgNPs}$ 催化剂, 而分离后的 $\mathrm{AgNPs}$ 进一步纯 化后可能可应用于医疗卫生领域.

上述结果表明 nZVI 富集 $\mathrm{Ag}^{+}$的过程中, $\mathrm{Ag}^{+}$被还原 为单质银并以球形纳米颗粒的形式附着在 nZVI 颗粒表 面, 简单地使用稀硫酸溶解含铁固体和聚乙烯吡咯烷酮 K-30(PVP)分散之后，即可获得 AgNPs.
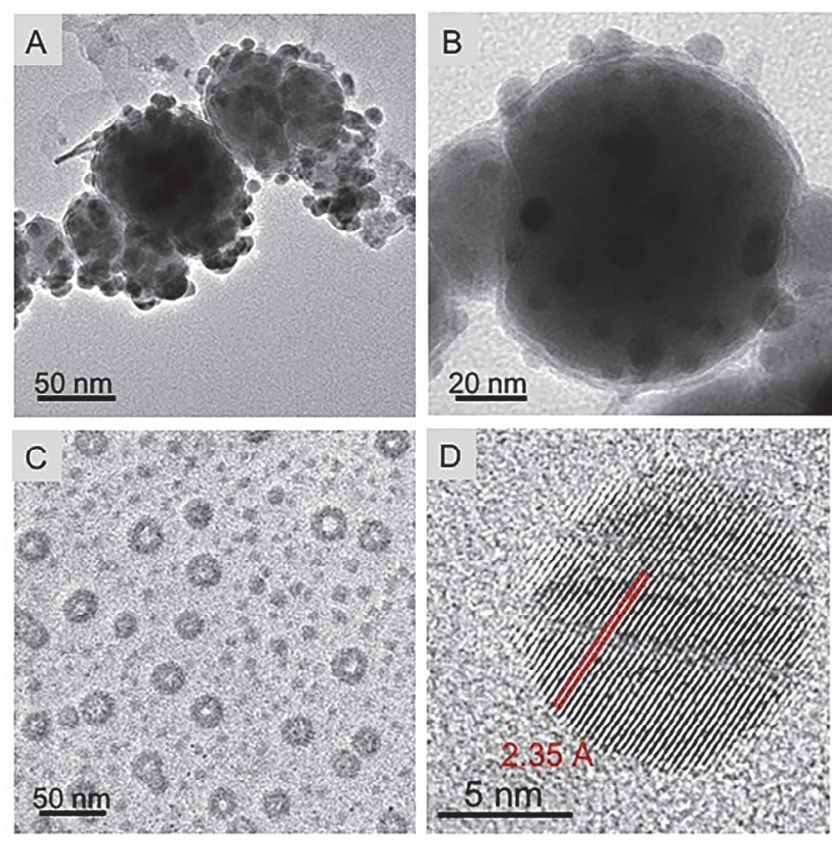

图 8 (A, B)反应产物 HR-TEM 图像; (C, D) PVP 和稀 $\mathrm{H}_{2} \mathrm{SO}_{4}$ 处理后产 物 HR-TEM 图像

Figure 8 (A, B) HR-TEM images of products; (C, D) HR-TEM images of products after $\mathrm{PVP}$ and $\mathrm{H}_{2} \mathrm{SO}_{4}$ treatment

Reaction conditions: $\left[\mathrm{Ag}^{+}\right]_{0}=1000 \mathrm{mg} / \mathrm{L}, \mathrm{nZVI}$ dosage $=1.0 \mathrm{~g} / \mathrm{L}$, initial $\mathrm{pH}=$ 5.0 , rotating speed $=200 \mathrm{r} / \mathrm{min}, 2{ }^{\circ} \mathrm{C}$

\section{3 结论}

本文研究并证实 “反应-分离-回用” 式 NIR 工艺能 够高效富集低浓度 $\mathrm{Ag}^{+}$(约 $1 \mathrm{mg} / \mathrm{L}$ ). 反应产物 Ag-nZVI 中 $\mathrm{Ag}$ 的含量可达 $32.0 \mathrm{mg} / \mathrm{g}$, 可解决分散银资源富集回 收的问题; $E_{\mathrm{h}}$ 调控机制稳定可靠, 可反映 NIR 富集效果, 将出水 $\mathrm{Ag}^{+}$浓度控制在较低水平. 相比于其他常用的吸 附/还原材料, nZVI 富集效率及速率高, 受 $\mathrm{pH}$ 影响小, 通过吸附、还原作用对 $\mathrm{Ag}$ 的最大富集负荷可达 5.3 g-Ag/g-nZVI. 使用 PVP 和稀硫酸处理反应后 nZVI 颗粒 即可得到高结晶度的球形 $(10 \mathrm{~nm})$ 纳米银 $(\mathrm{AgNPs})$ 单质.

\section{4 实验部分}

\section{$4.1 \mathrm{nZVI}$ 制备}

采用经典液相还原法 ${ }^{[15]}$, 即将 $\mathrm{NaBH}_{4}(0.2 \mathrm{~mol} / \mathrm{L})$ 逐 滴加至 $\mathrm{FeCl}_{3}(0.05 \mathrm{~mol} / \mathrm{L})$ 溶液中制备得到 $\mathrm{nZVI}$ [式(3)]:

$4 \mathrm{Fe}^{3+}+3 \mathrm{BH}_{4}^{-}+9 \mathrm{H}_{2} \mathrm{O} \rightarrow 4 \mathrm{Fe}^{0}+3 \mathrm{H}_{2} \mathrm{BO}_{3}^{-}+12 \mathrm{H}^{+}+6 \mathrm{H}_{2} \uparrow$

新鲜制备的 nZVI 使用去离子水和无水乙醇多次洗 涤后低温 $\left(4{ }^{\circ} \mathrm{C}\right)$ 保存于无水乙醇中.

\section{2 连续流实验}

纳米零价铁连续流反应器(图 1)运行参数如表 S2 所 示. 反应过程中在预设时间点取沉淀池出水, 过 0.22 $\mu \mathrm{m}$ 水相滤膜后加入 $\mathrm{HNO}_{3}(4 \%, V / V)$ 酸化, 测定溶解态 $\mathrm{Ag} 、 \mathrm{Fe}$ 离子浓度. 反应结束后取反应区 $\mathrm{nZVI}$ 混合物真 
空干燥后称重, 经 $\mathrm{HNO}_{3}$ 加热消解后使用电感耦合等离 子发射光谱仪(ICP-OES)测定其中 $\mathrm{Ag}$ 的固含量. 同时监 控反应区内 $E_{\mathrm{h}}$ 的变化.

\section{3 批次实验}

本部分研究了材料种类、溶液浓度、初始 $\mathrm{pH}$ 及 nZVI 投加量对 $\mathrm{Ag}^{+}$富集的影响.

研究不同材料的影响时, 向 $50 \mathrm{~mL}$ 玻璃瓶中加入 40 $\mathrm{mL} \mathrm{AgNO}_{3}$ 溶液 $\left(\left[\mathrm{Ag}^{+}\right]=100 \mathrm{mg} / \mathrm{L}, \mathrm{pH}=5.0\right)$, 通入高纯 氮气 $(>99.999 \%) 30 \mathrm{~min}$ 后, 分别加入 $0.04 \mathrm{~g}$ 固体材料, 放置于恒温摇床中 $\left(25{ }^{\circ} \mathrm{C}, 200 \mathrm{r} / \mathrm{min}\right)$ 反应 $4 \mathrm{~h}$. 分离系数 $K$ 计算公式如式(4)所示:

$$
K=C_{\mathrm{Ag}, \mathrm{s}} / C_{\mathrm{Ag}, \mathrm{a}}
$$

式中 $K(\mathrm{~L} / \mathrm{kg})$ 代表分离系数, $C_{\mathrm{Ag}, \mathrm{a}}(\mathrm{mg} / \mathrm{L})$ 和 $C_{\mathrm{Ag}, \mathrm{s}}(\mathrm{mg} / \mathrm{kg})$ 分别代表溶液中初始 $\mathrm{Ag}^{+}$浓度以及富集后固相上 $\mathrm{Ag}$ 的 含量.

研究溶液初始 $\mathrm{pH}$ 对 nZVI、mZVI 富集 $\mathrm{Ag}^{+}$(100 $\mathrm{mg} / \mathrm{L})$ 的影响时, 使用 $\mathrm{HNO}_{3}(0.1 \mathrm{~mol} / \mathrm{L})$ 和 $\mathrm{NaOH}(0.1$ $\mathrm{mol} / \mathrm{L})$ 将初始 $\mathrm{pH}$ 调节至 3.0、4.0、5.0、6.0、7.0、8.0、 9.0 , 后续实验步骤同上.

研究溶液初始浓度对 nZVI 富集 $\mathrm{Ag}^{+}$的影响时, 在 一系列浓度梯度 $\mathrm{AgNO}_{3}$ 溶液(初始 $\mathrm{pH}=5.0$ ) 中加入 1.0 $\mathrm{g} / \mathrm{L}$ nZVI，其余步骤同上.

研究 nZVI 投加量对其富集 $\mathrm{Ag}^{+}$的影响时, 在多口 烧瓶中加入 $1000 \mathrm{~mL} \mathrm{AgNO}_{3}$ 溶液 $\left(\left[\mathrm{Ag}^{+}\right]=1000 \mathrm{mg} / \mathrm{L}\right.$, 初始 $\mathrm{pH}=5.0$ ), 通入高纯氮气 $30 \mathrm{~min}$ 后, 分别投加 $0.10 、 0.25 、 0.50 、 1.00 \mathrm{~g} \mathrm{nZVI}$ ，间隔一定时间取样测定 混合液中剩余 $\mathrm{Ag}^{+}$浓度.

本部分所有实验均重复三次, 图中显示数据为三次 平行实验的算术平均值.

\section{4 固相表征}

$\mathrm{nZVI}$ 与 $\mathrm{AgNO}_{3}$ 反应后的固相产物分为两份. 其中 一份样品不做任何前处理, 即 Ag-nZVI, 另一份样品使 用 PVP (4 g/L) 和过量稀硫酸处理, 所用稀硫酸体积比 $20 \%$, PVP 浓度 $4 \mathrm{~g} / \mathrm{L}$, 恒温 $\left(60{ }^{\circ} \mathrm{C}\right)$ 水浴加热后离心, 取 底部沉淀，保存在 $4 \mathrm{~g} / \mathrm{L}$ PVP 中. 采用 HR-TEM、XRD、 XPS 分析相关材料形貌、物相及表面 $\mathrm{Ag}$ 的价态.

\section{References}

[1] Yu, S. L.; Yin, Y. G.; Liu, J. F. Environ. Sci.-Proc. Imp. 2013, 15, 78.

[2] Syed, S. Waste. Manage. 2016, 50, 234.

[3] World Silver Survey, 2017, GFMS Limited/The Silver Institute. $\mathrm{http}: / /$ www.silverinstitute.org

[4] Benn, M. T.; Westerhoff, P. Environ. Sci. Technol. 2008, 42, 4133.

[5] Zhou, X. X.; Liu, J. F.; Yuan, C. G.; Chen, Y. S. J. Anal. Atom. Spec- trom. 2016, 31, 2285.

[6] Eckelman, M. J.; Graedel, T. E. Environ. Sci. Technol. 2007, 41, 6283.

[7] Li, R.; Lu, Y. Y.; Lei, K. X.; Li, F. J.; Cheng, F. Y.; Chen, J. Acto Chim. Sinica 2017, 75, 199 (in Chinese). (李再, 卢艳莹, 雷凯翔, 李福军, 程方益, 陈军, 化学学报, 2017, 75, 199.)

[8] Wang, C.; Deng, N.; Wang, L. L.; Xu, D. J.; Yao, X. Q. Chinese J. Org. Chem. 2016, 36, 1034 (in Chinese). (王超, 邓楠, 王玲玲, 许 定健, 姚小泉, 有机化学, 2016, 36, 1034.)

[9] Vance, M. E.; Kuiken, T.; Vejerano, E. P.; McGinnis, S. P.; Hochella, M. F.; Rejeski, D.; Hull, M. S. Beilstein. J. Nanotech. 2015, 6, 1769.

[10] Song, X. H.; Gunawan, P.; Jiang, R. R.; Leong, S. S. J.; Wang, K.; Xu, R. J. Hazard. Mater. 2011, 194, 162

[11] Zhou, Y. M.; Gao, B.; Zimmerman, R. A.; Cao, X. D. Chemosphere 2014, 117,801 .

[12] Celik. Z.; Gulfen. M.; Aydin, A. O. J. Hazard. Mater. 2010, 174, 556.

[13] Wang, H. Y.; Gao, H.; Sun, J. S.; Li, J.; Su, Y. X.; Ji, Y. L.; Gong, C. M. Desalination 2011, 270, 258

[14] Huo, H. Y.; Su, H. J.; Tan, T. W. Chem. Eng. J. 2009, 150, 139.

[15] Huang, X. Y.; Wang, W.; Ling, L.; Zhang, W. X. Acta Chim. Sinica 2017, 75, 529 (in Chinese). (黄潇月, 王伟, 凌岗, 张伟贤, 化学学 报, 2017, 75, 529.)

[16] Mu, Y.; Jia, F. L.; Ai, Z. H.; Zhang, L. Z. Environ. Sci.-Nano 2017, $4,27$.

[17] Fu, F. L.; Dionysiou, D. D.; Liu, H. J. Hazard. Mater. 2014, 267, 194.

[18] Zhang, Y. L.; Yan, J.; Dai, C. M.; Li, Y. T. ; Zhou, Y.; Zhou, X. F. J. Nanopart. Res. 2015, 17, 1110.

[19] Teng, W.; Fan, J. W.; Wang, W.; Bai, N.; Liu, R.; Liu, Y.; Deng, Y. H.; Kong, B.; Yang, J. P.; Zhao, D. Y.; Zhang, W. X. J. Mater. Chem. A 2017, 5, 4478 .

[20] Ling, L.; Zhang, W. X. J. Am. Chem. Soc. 2015, 137, 2788.

[21] Sheng, G. D.; Yang, P. J.; Tang, Y. N.; Hu, Q. Y.; Li, H.; Ren, X. M.; Hu, B. W.; Wang, X. K.; Huang, Y. Y. Appl. Catal. B-Environ. 2016, $193,189$.

[22] Xia, X. F.; Hua, Y. L.; Huang, X. Y.; Ling, L.; Zhang, W. X. Acta Chim. Sinica 2017, 75, 594 (in Chinese). (夏雪芬, 滑熠龙, 黄潚 月, 凌岗, 张伟贤, 化学学报, 2017, 75, 594.)

[23] Sheng, G. D.; Alsaedi, A.; Shammakh, W.; Monaquel, S.; Sheng, J.; Wang, X. K.; Li, H.; Huang, Y. Y. Appl. Carbon. 2016, 99, 123.

[24] Li, S. L.; Wang, W.; Liu, Y. Y.; Zhang, W. X. Chem. Eng. J. 2014, $254,115$.

[25] Wang, W.; Hua, Y. L.; Li, S. L.; Yan, W. L.; Zhang, W. X. Chem. Eng. J. 2016, 304, 79

[26] Li, S. L.; Wang, W.; Liang, F. P.; Zhang, W. X. J. Hazard. Mater. 2017, 322, 163 .

[27] Wang, W.; Li, S. L.; Lei, H.; Pan, B. C.; Zhang, W. X. Chem. Eng. J. 2015, 260, 616 .

[28] Shi, Z. Q.; Nurmi, T. J.; Tratnyek, G. P. Environ. Sci. Technol. 2011, $45,1586$.

[29] Sverdrup, H.; Koca, D.; Ragnarsdottir, V. K. Resour. Conserv. Recy. 2014, 83, 121.

[30] Liang, L. P.; Yang, W. J.; Guan, X. H.; Li, J. L.; Xu, Z. J.; Wu, J.; Huang, Y. Y.; Zhang, X. Z. Water Res. 2013, 47, 5846.

[31] Guan, X. H.; Sun, Y. K.; Qin, H. J.; Li, J. X.; Lo, I. M. C.; He, D.; Dong, H. R. Water Res. 2015, 75, 224.

[32] Liang, L. P.; Sun, W.; Guan, X. H.; Huang, Y. Y.; Choi, W. Y.; Bao, H. L.; Li, L. N.; Jiang, Z. Water Res. 2014, 49, 371.

[33] Nitayaphat, W.; Jintakosol, T. J. Clean. Prod. 2015, 87, 850.

[34] Wang, Y.; Ma, X. J.; Li, Y. F.; Li, X. L.; Yang, L. Q.; Ji, L.; He, Y. Chem. Eng. J. 2012, 209, 394.

[35] Ju, S. H.; Zhang, Y. F.; Zhang, Y.; Xue, P. Y.; Wang, Y. H. J. Hazard. Mater. 2011, 192, 554.

[36] Yin, Y. G.; Shen, M. H.; Tan, Z. Q.; Yu, S. J.; Liu, J. F.; Jiang, G. B. Environ. Sci. Technol. 2015, 49, 6581 . 